\title{
INEQUALITIES FOR ANGLES BETWEEN SUBSPACES \\ WITH APPLICATIONS TO CAUCHY-SCHWARZ INEQUALITY IN INNER PRODUCT SPACES
}

\section{ZDZISEAW OTACHEL}

Abstract. We show several inequalities for angles between vectors and subspaces in inner product spaces, where concave functions are involved. In specific situations, some of them can be interpreted as triangle inequalities for natural metrics on complex projective spaces. In a consequence, we obtain a few operator generalizations of the famous Cauchy-Schwarz inequality, where powers grater than two occur. Mathematics subject classification (2010): 46C05, 15A455.

Keywords and phrases: Angle between vectors/subspaces, triangle inequality, Krein's inequality, CauchySchwarz's inequality, orthogonal projection, Gram determinant.

\section{REFERENCES}

[1] J. M. Aldaz, S. BarZa, M. FujiI, M. S. Moslehian, Advanced in operator Cauchy-Schwarz inequalities and their reverses, Ann. Funct. Anal. 6 (2015) (3), 275-295.

[2] F. Deutsch, The angle between subspaces of a Hilbert space, Approximation Theory, Wavelets and Applications, Singh S (ed.). Kluwer: Dordrecht, (1995) 107-130.

[3] J. DiXMIER, Étude sur less variétés et les opératerus de Julia avec quelques applications, Bull. Soc. Math. France 77 (1949), 11-101.

[4] S. S. Dragomir, A survey on Cauchy-Bunyakovsky-Schwarz type discrete inequalities, J. Inequal. Pure Appl. Math. 4 (3) (2003), Article 63.

[5] S. S. DRAgomir, Advances in Inequalities of the Schwarz, Grüss and Bessel Type in Inner Product Spaces, Nova Science Publishers, New York 2005.

[6] S. S. DRAGOMIR, Advances in Inequalities of the Schwarz, Triangle and Heisenberg Type in Inner Product Spaces, Nova Science Publishers, New York 2007.

[7] S. S. DRAGOMIR, Operator refinements of Schwarz inequality in inner product spaces, doi: 10.1080/03081087.2018.1472204, Linear Multilinear Algebra (2018).

[8] S. S. DRAGOMIR, Improving Schwarz inequality in inner product spaces, Linear Multilinear Algebra 67 (2) $(2019$,$) 337-347.$

[9] K. FRIEDRICHS, On certain inequalities and characteristic value problems for analytic functions and for functions of two variables, Trans. Amer. Math. Soc. 41 (1937), 321-364.

[10] T. FURUTA, An elementary proof of Hadamard theorem, Math. Vesnik 8 (23) (1971), 267-269.

[11] M. KREIN, Angular localization of the spectrum of a multiplicative integral in a Hilbert space, Funct. Anal. Appl. 3 (1969), 89-90.

[12] M. Lin, Remarks on Krein's inequality, Math. Intell. 34 (1) (2012), 3-4.

[13] D. S. Mitrinović, J. E. PeČARIĆ, A. M. Fink, Classical and New Inequalities in Analysis, Kluwer Academic Publishers, Dordrecht/Boston/London (1993).

[14] J. M. Steele, The Cauchy-Schwarz Master Class, Cambridge University Press, NY (2004).

[15] S. G. WALKER, A self-improvement to the Cauchy-Schwarz inequality, Stat. Probab. Lett. 122 (2017), 86-89.

[16] B. WANG AND F. ZHAng, A trace inequality for unitary matrices, Amer. Math. Monthly 101 (1994), 453-455. 